\title{
Midline fascial plication under continuous digital transrectal control: which factors determine anatomic outcome?
}

\author{
Alfredo L. Milani • Mariella I. J. Withagen • \\ Karlijn J. Schweitzer • Erica W. M. Janszen • \\ Mark E. Vierhout
}

Received: 18 August 2009/Accepted: 31 December 2009 / Published online: 10 February 2010

(C) The Author(s) 2010. This article is published with open access at Springerlink.com

\begin{abstract}
Introduction and hypothesis The aim of the study was to report anatomic and functional outcome of midline fascial plication under continuous digital transrectal control and to identify predictors of anatomic failure.

Methods Prospective observational cohort. Anatomic success defined as POP-Q stage $\leq \mathrm{I}$ of the posterior compartment. Validated questionnaires to measure bother and impact on quality of life. Logistic regression to identify risk factors for anatomic failure.

Results Two hundred thirty-three patients with posterior pelvic organ prolapse (POP) stage $\geq$ II underwent midline fascial plication under continuous digital transrectal control. Median follow-up was 14 months (12-35 months), and anatomic success was $80.3 \%$ (95\% CI 75-86). Independent predictors of failure were posterior compartment POP stage $\geq$ III [OR 8.7 (95\% CI 2.7-28.1)] and prior colposus-
\end{abstract}

\section{A. L. Milani $(\bowtie)$}

Department of Obstetrics and Gynaecology,

Reinier de Graaf Group,

PO Box 5011, 2600 GA Delft, The Netherlands

e-mail: almilani@telfort.nl

M. I. J. Withagen · M. E. Vierhout

Department of Obstetrics and Gynaecology,

Radboud University Nijmegen Medical Centre,

PO Box 9101, 6500 HB Nijmegen, The Netherlands

\section{K. J. Schweitzer}

Department of Obstetrics and Gynaecology,

University Medical Centre,

PO Box 85500, 3508 GA Utrecht, The Netherlands

E. W. M. Janszen

Department of Obstetrics and Gynaecology,

Onze Lieve Vrouwe Gasthuis,

P.O. Box 95500, 1090 HM Amsterdam, The Netherlands pension [OR 5.6 (95\% CI 1.1-27.8)]. Sixty-three percent of patients bothered by obstructed defaecation experienced relief after surgery.

Conclusions Anatomic and functional outcomes were good. Risk factors for anatomic failure were initial size of posterior POP (stage $\geq$ III) and prior colposuspension.

Keywords Anatomic $\cdot$ Colposuspension - Continuous digital transrectal control $\cdot$ Midline fascial plication . Posterior wall $\cdot$ Prolapse repair
Abbreviations
POP-Q Pelvic organ prolapse quantification
UDI Urogenital distress inventory
DDI Defaecatory distress inventory
IIQ Incontinence impact questionnaire
POP Pelvic organ prolapse

\section{Introduction}

Posterior colporrhaphy is reported to be one of the most common gynaecological procedures performed in over $40 \%$ of women undergoing surgical correction of prolapse [1].

Restoration of the posterior compartment, which includes perineum, rectum, and the peritoneum of the culde-sac, knows many approaches. The transvaginal, transanal, and laparoscopic approaches have been described to correct defects in this compartment [2].

In a randomised controlled trial that compared the transanal with the transvaginal approach, the latter approach proved to be anatomically more successful [3]. Maher et al. have demonstrated excellent anatomic and functional outcomes after midline rectovaginal fascial plication [4]. In a 
retrospective survey by Abramov et al., a significant higher anatomic recurrence rate of rectoceles was noted after discrete site-specific repair as compared with the midline fascial plication [5]. Therefore, at present, the midline plication of rectovaginal connective tissue is considered to be the most suitable surgical approach for the repair of symptomatic posterior vaginal wall prolapse.

We have added, in our opinion, an important element of the discrete fascial defect repair to our surgical protocol of midline fascial plication, namely the continuous digital transrectal control with the index finger of the nondominant hand [6].

In the majority of women, pelvic organ support defects rarely exist in only one vaginal compartment; in a population-based sample of women with POP, the most frequent single compartment affected was the posterior wall, where isolated prolapse was seen in $20 \%$ of women, but the most common prolapse combination was anterior and posterior wall in 40\% [7]. Therefore, combined surgical procedures will often have to be performed to correct these defects. The influence of concomitant POP surgery on the anatomic outcome in the posterior compartment has not received much attention in the literature [8].

The aim of this article is twofold: to report the anatomical and functional outcome of midline fascial plication under continuous digital transrectal control and to identify independent risk factors for anatomic failure in the posterior compartment.

\section{Material and methods}

In 2003, we started a prospective data registry of all patients undergoing POP surgery in two major urogynecological centres in The Netherlands, the Reinier de Graaf Hospital in Delft and the Radboud University Medical Centre in Nijmegen. After obtaining informed consent, consecutive patients were enrolled in this prospective observational cohort registry.

Preoperatively, genital prolapse was quantified in the dorsal lithotomy position using the pelvic organ prolapse quantification (POP-Q) measurement system, as recommended by the International Continence Society (ICS) [9]. Patients were asked to complete the standard urogynecological questionnaire of the Dutch Pelvic Floor Society.

This questionnaire contains the validated Dutch versions of the urinary distress inventory (UDI), the Incontinence Impact Questionnaire (IIQ) and the Defaecatory Distress Inventory (DDI) [10-12]. The questionnaire contains some questions on sexual functioning as well. Patients rate the amount of bother in various domains on a 5-point Likert scale, ranging from 0 (no bother at all) to 4 (a lot of bother). Domain scores for UDI, DDI and IIQ are calculated and range from 0 (no bother at all or best quality of life) to100 (most bother or worst quality of life). Patients were considered to be significantly bothered in any of the DDI domains if the average answer to the questions of that particular domain was at least a little bother (to a lot), which was equivalent to a domain score of $\geq 33$ (range, 0-100).

\section{Surgical procedure}

Peroperative antibiotic prophylaxis was given with a single dose of cefalozine-natrium (Kefzol ${ }^{\circledR}$, Lilly, The Netherlands) and metronidazol (Flagyl ${ }^{\circledR}$, Aventis Pharma BV Hoevelaken, The Netherlands). Patients were positioned in the dorsal lithotomy position with their hips flexed between $90^{\circ}$ and $110^{\circ}$. After hydrodissection (lidocaine hydrochloride monohydrate $200 \mathrm{mg}$ with epinephrine hydrogen tartrate $100 \mu \mathrm{g}$ in $20 \mathrm{ml}$-Astra Zeneca BV Zoetermeer the Netherlands-diluted in $100 \mathrm{ml}$ of $0.9 \%$ saline solution) a midline incision was made in the posterior vaginal wall from an area at least $1 \mathrm{~cm}$ above the superior aspect of the vaginal defect close to the posterior fornix all the way to the level of the posterior fourchette. The incision was not as deep as is used in mesh surgery, but at a more superficial level to allow identification of the so-called rectovaginal "fascia", achieved by cleaving the vaginal wall at the level of its fibromuscular and adventitial layer. Allis clamps were placed on the vaginal walls, and usually, gentle sideward traction produced a nice cleavage area. A gloved finger covered with an unwound gauze helped further blunt dissection when considered necessary. The gloved index finger of the non-dominant hand was then introduced into the rectum, and with the finger and thumb of this hand, connective tissue could be grasped on both lateral sides to allow placement of several interrupted Vicryl 2-0 sutures. More cranially, the connective tissue layer is less apparent, and at these points, a thin layer of the adventitia and fibromuscular vaginal wall was grasped bilaterally to be incorporated in the sutures [13]. Plication of the fascia was performed in a cranial-caudal direction with an average number of 6-8 sutures. Knots of the sutures were tied by the assistant under continuous control by the nondominant finger of the surgeon. On removal of the finger from the rectum, gloves were changed, and modest vaginal trimming was performed bilaterally. A running Vicryl 2-0 suture was used to close the vaginal wall from cranial to caudal direction (Fig. $1 \mathrm{a}-\mathrm{h}$ ). A gauze pack was left overnight in the vagina as well as an indwelling urinary catheter. Patients were all operated by or under supervision of the first or last author. The other authors performed surgery after being trained by the former. 

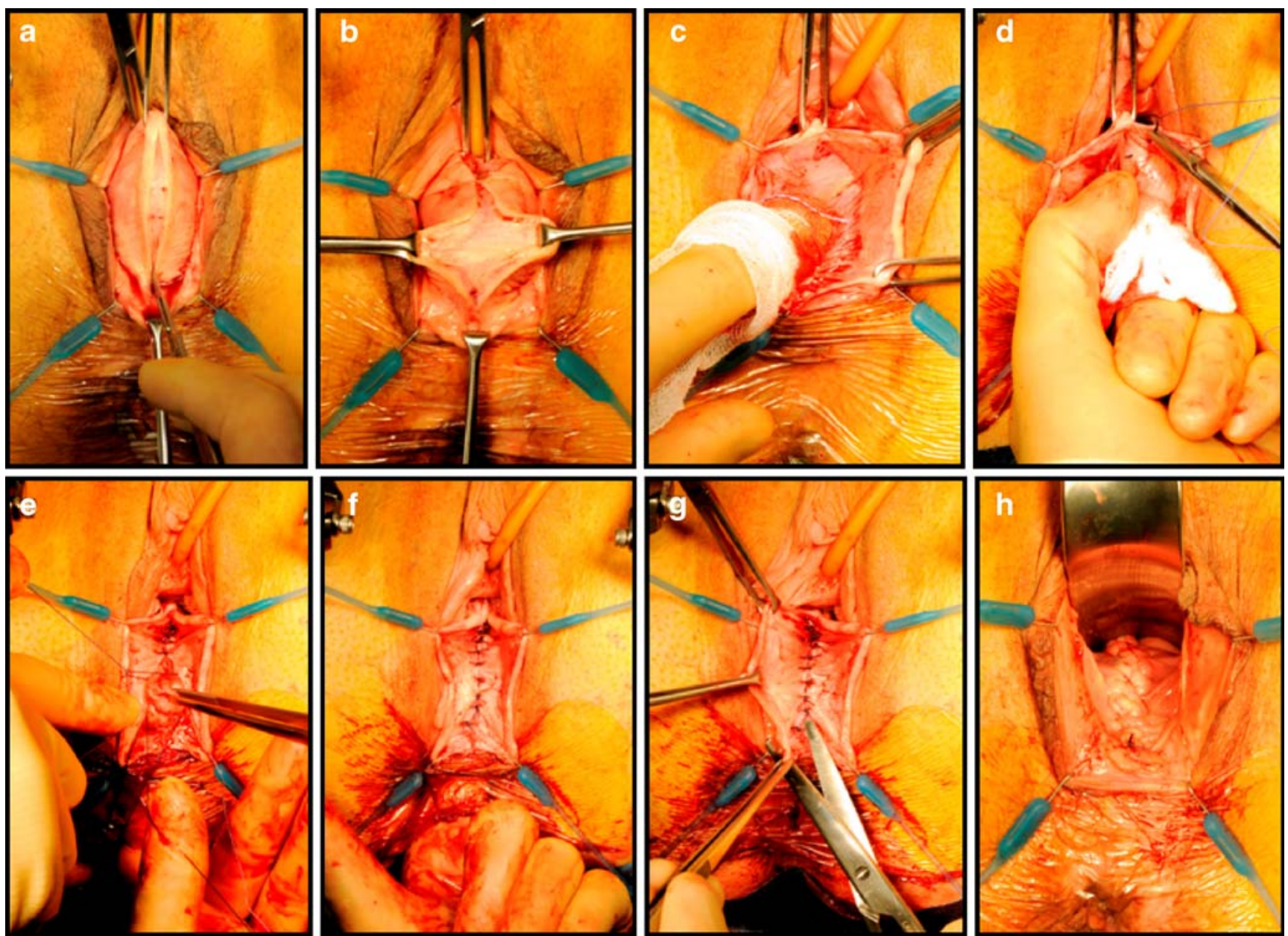

Fig. 1 Midline fascial plication under continuous transrectal control of a stage II posterior vaginal wall prolapse. a Incision after hydrodissection. b Sideward traction and cleaving of vaginal wall. $\mathbf{c}$ Blunt dissection of

fascia. d Suturing at cranial side of rectocele. e Tie of a knot by the assistant of the surgeon. f Rectal control of firmness. $\mathbf{g}$ Trimming of vaginal wall. $\mathbf{h}$ End result after running suture of vaginal wall

\section{Study endpoints}

Primary endpoints of this study were anatomic outcome of the posterior compartment after a follow-up period of at least 12 months and the identification of independent predictors of anatomic failure. Secondary endpoints were functional efficacy in terms of significant change in experienced bother in the various domains of UDI, DDI and IIQ, as well as effect of surgery on dyspareunia.

Anatomic success was defined as ICS POP stage 0 or I of the posterior compartment. Anatomic failure is a POP stage $\geq$ II of the posterior compartment [9]. Follow-up investigations were performed, by the second, third and fourth author.

\section{Data collection}

To obtain data on anatomical efficacy POP-Q, measurements at baseline were compared with those obtained at follow-up. Data on the functional efficacy, quality of life

and effect on sexual function were derived from the standard urogynecological questionnaires at baseline and follow-up. Data were analysed using a Statistical Package for the Social Sciences 17.0 database.

Baseline and surgical data are presented as median (range) or numbers with corresponding percentages and where appropriate, with $95 \%$ confidence intervals. Pearson's Chi-square was used where appropriate. Domain scores of UDI, DDI and IIQ are presented as means with standard deviations. Differences in means between baseline and follow-up were tested with the paired-sample $t$ test. A $p \leq 0.05$ was considered significant. To quantify clinically relevant effects, effect sizes were calculated in the various domains of UDI, DDI and IIQ by using Cohen's $d$, which represents the difference between two means divided by the pooled standard deviations of these means. The effect size is defined as small if Cohen's $d=0.2$, medium if $d=0.5$ and large if $d \geq 0.8$ [14].

Univariate logistic analysis was performed to select potential risk factors for anatomic failure. Covariates that 
showed a $p<0.1$ at univariate analysis were entered in a stepwise forward multivariate logistic regression model to identify independent predictors of anatomic failure. $P$ values $<0.05$ were considered significant, and data are presented as adjusted odds ratio with $95 \%$ confidence intervals.

\section{Results}

Two hundred and thirty-three patients with a posterior vaginal wall prolapse stage II or more were operated and available for follow-up analysis. Median follow-up was 14 months (12-35 months). Not all patients responded to our invitation for follow-up examination, but on 208 (89\%) patients, a POP-Q examination could be performed. At baseline, one hundred and eighty-seven $(80 \%)$ and at follow-up two hundred and fourteen patients (92\%) responded to our request to complete the urogynecological questionnaire.

Baseline and surgical characteristics are presented in Table 1. Seventy-three patients (31\%) underwent an isolated posterior wall repair procedure; all others $(69 \%)$ underwent concomitant repairs in the other vaginal compartments as well.

\section{Anatomical results}

Data on POP-Q measurements and POP stage at baseline and follow-up are shown in Table 2.

Overall anatomic success in the posterior compartment was $80 \%$ (95\% CI 75-86).

Sub-analysis revealed a success rate of $85 \%(95 \%$ CI 79-91) for combined posterior repairs and 70\% (95\% CI 59-81) for isolated repairs.

\section{Predictors of failure}

Table 3 shows the results of univariate and multivariate analysis of covariates potentially related to anatomic failure. Five covariates were entered in a stepwise forward multivariate logistic analysis model. Posterior POP stage $\geq$ III and a history of prior colposuspension were the only factors that could be identified as independent significant predictors of anatomic failure in the posterior compartment.

Functional results

Functional data on the various domains of UDI, DDI and IIQ with their respective calculated effect sizes are summarised in Table 4. Except for faecal incontinence all domains of UDI, DDI and IIQ showed statistically significant improvements. The domains of genital prolapse
Table 1 Baseline and surgical characteristics

\begin{tabular}{lc}
\hline & $n=233$ \\
\hline Age, years & $59(29-85)$ \\
BMI. kg/m & $25(16-42)$ \\
Parity & $2(1-10)$ \\
Postmenopausal & $180(77 \%)$ \\
& Prior related surgery \\
Vaginal hysterectomy & $77(33 \%)$ \\
Abdominal hysterectomy & $39(17 \%)$ \\
Anterior colporrhaphy & $51(22 \%)$ \\
Posterior colporrhaphy & $44(19 \%)$ \\
Sacrocolpopexy & $12(5 \%)$ \\
2 prior prolapse repairs & $21(9 \%)$ \\
$\geq 3$ prior prolapse repairs & $24(10 \%)$ \\
Colposuspension & Prior incontinence surgery \\
TVT & $34(15 \%)$ \\
Isolated posterior repair & $8(3 \%)$ \\
Combined repairs & Surgical procedures \\
Anterior colporrhaphy & $73(31 \%)$ \\
Perineoplasty & $160(69 \%)$ \\
Manchester procedure & $143(89 \%)$ \\
Vaginal hysterectomy & $38(24 \%)$ \\
McCall and enteroceleplasty & $28(17 \%)$ \\
Hospital stay, days & $43(27 \%)$ \\
\hline & $22(14 \%)$ \\
Bloodloss, ml) & Surgical characteristics \\
General anaesthesia & $75(10-205)$ \\
& $100(40-600)$ \\
& $104(45 \%)$ \\
& $129(55 \%)$ \\
& $4-13)$ \\
\hline
\end{tabular}

Data presented as median (range) or number (percentages)

and pain in the UDI revealed a large treatment effect size (Cohen's $d \geq 0.8$ ). For all DDI domains, effect sizes appeared to be of medium size only (0.3-0.7).

At baseline, 150 patients completed all questions in the domain of obstructed defaecation. Thirty-eight $(25 \%)$ were considered to be significantly bothered (domain score $\geq 33$ ). Mean domain score before surgery of these patients was 48.5 (SD, 16.9). Mean postoperative score was 22.5 (SD, 17.2; $p<0.001$, effect size: 1.5 ). Twenty-four patients (63\%) reported to be cured or improved at follow-up.

At baseline, 170 of the 187 patients who completed the urogynecological questionnaire answered questions on sexual functioning. One hundred ten patients (65\%) reported to be sexually active. At follow-up, this percentage remained unchanged, $64 \%$. At baseline, $42 \%$ of patients reported to have some degree of dyspareunia. At follow-up, this rate had dropped to $34 \%$. Nineteen percent of patients 
Table 2 POP stage and POP-Q variables at baseline and follow-up
Data are presented as mean ( \pm standard deviation) $\mathrm{cm}$ for POP-Q variable and as number of patients (percentage) for POP stage

$\mathrm{Ba}$ most descendant point at anterior vaginal wall. $C$ vaginal apex. $B p$ most descendant point at posterior vaginal wall

(All in centimetre distance from the hymenal remnants)

TVL total vaginal length, $G H$ genital hiatus $\mathrm{PB}$, perineal body length in centimetres $( \pm$ standard deviation)

${ }^{\text {a }}$ 95\% Confidence interval

${ }^{\mathrm{b}}$ Pearson's Chi-square: $p<0.001$

\begin{tabular}{|c|c|c|c|c|c|c|}
\hline \multicolumn{2}{|c|}{ POP-Q variable } & \multicolumn{2}{|l|}{ Baseline (233) } & \multicolumn{2}{|c|}{ Follow-up (208) } & Change from baseline \\
\hline \multicolumn{2}{|l|}{$\mathrm{Ba}$} & \multicolumn{2}{|l|}{$0.2(2.2)$} & \multicolumn{2}{|l|}{$-1.5(1.5)$} & $1.7(2.2)$ \\
\hline \multicolumn{2}{|l|}{$\mathrm{C}$} & \multicolumn{2}{|l|}{$-4.2(3.4)$} & \multicolumn{2}{|l|}{$-6.7(2.3)$} & $2.5(4.1)$ \\
\hline \multicolumn{2}{|l|}{$\mathrm{D}$} & \multicolumn{2}{|l|}{$-5.2(3.3)$} & \multicolumn{2}{|l|}{$-7.8(2.2)$} & $1.4(4.0)$ \\
\hline \multicolumn{2}{|l|}{ Bp } & \multicolumn{2}{|l|}{$0.5(1.2)$} & \multicolumn{2}{|l|}{$-2.2(1.2)$} & $2.7(1.3)$ \\
\hline \multicolumn{2}{|l|}{ TVL } & \multicolumn{2}{|l|}{$9.4(1.5)$} & \multicolumn{2}{|l|}{$9.1(0.9)$} & $0.3(1.6)$ \\
\hline \multicolumn{2}{|l|}{ GH } & \multicolumn{2}{|l|}{$4.6(1.1)$} & \multicolumn{2}{|l|}{$3.7(0.9)$} & $0.8(1.2)$ \\
\hline \multicolumn{2}{|l|}{ PB } & \multicolumn{2}{|l|}{$3.4(1.2)$} & \multicolumn{2}{|l|}{$3.8(1.0)$} & $0.4(1.4)$ \\
\hline \multicolumn{6}{|l|}{ POP stage } & Success rate \\
\hline \multirow[t]{5}{*}{ Anterior } & 0 & \multicolumn{2}{|l|}{$26(11.1 \%)$} & \multirow{2}{*}{\multicolumn{2}{|c|}{$63(30.3 \%)$}} & $61.5 \%(54.4-67.7)$ \\
\hline & I & \multicolumn{2}{|l|}{$40(17.2 \%)$} & & $65(31.2 \%)$ & \\
\hline & II & \multicolumn{2}{|l|}{$98(42.1 \%)$} & \multicolumn{2}{|l|}{$75(36.1 \%)$} & \\
\hline & III & \multicolumn{2}{|l|}{$69(29.6 \%)$} & $5(2.4 \%)$ & & \\
\hline & IV & - & & - & & \\
\hline Apical & 0 & $55(23.6 \%)$ & & $87(41.8 \%)$ & & $97.6 \%(95.5-99.7)$ \\
\hline & I & $142(60.9 \%)$ & & $116(55.8 \%)$ & & \\
\hline & II & $17(7.3 \%)$ & & $1(0.5 \%)$ & & \\
\hline & III & $19(8.2 \%)$ & & $4(1.9 \%)$ & & \\
\hline & IV & - & & - & & \\
\hline Posterior & & Isolated & Combined & Isolated & Combined & $80.3 \%(74.9-85.7)^{\mathrm{a}}$ \\
\hline & 0 & - & - & $27(40.3 \%)$ & $80(56.7 \%)$ & \\
\hline & I & - & - & $20(29.8 \%)$ & $40(28.4 \%)$ & \\
\hline & II & $43(58.9 \%)$ & $136(85 \%)$ & $17(25.4 \%)$ & $21(14.9 \%)$ & \\
\hline & III & $30(41.1 \%)^{b}$ & $24(15 \%)^{b}$ & $3(4.5 \%)$ & - & \\
\hline & IV & - & - & - & - & \\
\hline
\end{tabular}

who did not complain of dyspareunia before surgery reported this complaint at follow-up, so this was considered to be de novo dyspareunia. Fifty-eight percent of patients, however, who initially complained of dyspareunia, reported to be cured at follow-up.

\section{Discussion}

The surgical technique we used actually is a combination of the classical midline "fascial" plication and a site defect specific repair. The addition of continuous transrectal

Table 3 Univariate and multivariate logistic regression analysis of factors that might influence anatomic outcome

\begin{tabular}{|c|c|c|c|c|}
\hline \multirow[b]{2}{*}{ Covariates } & \multicolumn{2}{|c|}{ Univariate analysis } & \multicolumn{2}{|c|}{ Multivariate analysis } \\
\hline & $p$ & OR $(95 \% \mathrm{CI})$ & $p$ & OR $(95 \% \mathrm{CI})$ \\
\hline Age & 0.089 & $1.026(0.996-1.058)$ & 0.409 & \\
\hline Prior posterior wall repair & 0.243 & $0.292(0.037-2.302)$ & & \\
\hline Prior sacrocolpopexy & 0.602 & $0.563(0.065-4.877)$ & & \\
\hline Prior POP surgery & 0.068 & $1.917(0.954-3.851)$ & 0.529 & \\
\hline Prior colposuspension & 0.062 & $3.893(0.934-16.226)$ & $0.037^{*}$ & $5.558(1.112-27.779)$ \\
\hline Isolated posterior repairs & $0.013^{*}$ & $2.432(1.209-4.892)$ & 0.124 & \\
\hline Concomitant anterior colporrhaphy & 0.439 & $0.711(0.934-16.226)$ & & \\
\hline Concomitant apical support surgery & 0.774 & $0.882(0.375-2.076)$ & & \\
\hline Concomitant perineoplasty & 0.198 & $0.497(0.171-1.440)$ & & \\
\hline Concomitant vaginal hysterectomy & 0.300 & $0.586(0.213-1.611)$ & & \\
\hline Posterior compartment stage $\geq \mathrm{III}$ & $<0.001^{*}$ & $7.613(3.585-16.170)$ & $<0.001^{*}$ & $8.767(2.739-28.060)$ \\
\hline
\end{tabular}

Data presented with $p$ values and odds ratio's (OR) with $95 \%$ CI

Covariates with $p<0.1$ in univariate analysis $(n=5)$ were entered in a multivariate logistic analysis model, method stepwise forward (LR)

Significant $p$ values are shown with an asterisk 
Table 4 UDI, DDI and IIQ domain scores at baseline and follow-up with calculated effect sizes
$U D I$ urinary distress inventory, $D D I$ defaecatory distress inventory, IIQ incontinence impact questionnaire

Scores presented as mean \pm standard deviation

Scores range between 0 (least bother and best quality of life) to 100 (maximum bother and worst quality of life)

$P^{*}$ Paired-sample $t$ test.

${ }^{\text {a }}$ Effect size (Cohen's $d$ ): small, 0.2 ; medium, 0.5 ; large $\geq 0.8$

\begin{tabular}{lcccc}
\hline Domains UDI & Baseline (187) & Follow-up (214) & $P^{*}$ & Effect size $^{\mathrm{a}}$ \\
\hline Prolapse & $45.1(33.0)$ & $7.7(18.5)$ & $<0.001$ & 2.9 \\
Incontinence & $26.5(26.1)$ & $20.9(23.7)$ & $<0.001$ & 0.5 \\
Overactive bladder & $31.7(24.9)$ & $22.9(23.2)$ & $<0.001$ & 0.7 \\
Obstructive micturition & $25.8(26.9)$ & $17.7(23.0)$ & $<0.001$ & 0.6 \\
Pain & $33.7(29.3)$ & $18.1(23.0)$ & $<0.001$ & 1.2 \\
Domains DDI & & & & 0.4 \\
Constipation & $16.8(20.4)$ & $12.2(19.3)$ & 0.002 & 0.7 \\
Obstructed defaecation & $17.5(20.6)$ & $11.2(15.1)$ & $<0.001$ & 0.4 \\
Pain & $14.6(23.0)$ & $10.1(20.0)$ & 0.014 & 0.3 \\
Incontinence & $7.1(16.0)$ & $5.8(14.2)$ & 0.065 & \\
Domains IIQ & & & & 0.8 \\
Physical functioning & $27.6(28.3)$ & $16.2(27.2)$ & $<0.001$ & 0.8 \\
Mobility & $33.0(25.7)$ & $22.3(24.8)$ & $<0.001$ & 0.7 \\
Emotional health & $27.1(26.3)$ & $17.9(24.5)$ & $<0.001$ & 0.4 \\
Social functioning & $16.1(18.3)$ & $12.0(19.3)$ & $<0.001$ & 0.3 \\
Embarrassment & $15.0(20.4)$ & $12.2(21.6)$ & 0.005 & \\
\hline
\end{tabular}

digital control during the procedure helps identify any interruptions in the connective tissue layers, and one ensures that the repair is sufficiently solid, has no weak spots and that no sutures accidentally enter the rectal lumen. A relative disadvantage of our technique though, is the fact that the surgeon cannot tie the sutures himself but has to rely on the assistant for this. Whether this protocol assignment significantly contributes to the anatomical efficacy is not shown by our study, but can only be determined in a controlled study that compares the "classical" midline plication with a procedure that adds this "continuous transrectal control".

\section{Anatomical results and risk factors for failure}

The overall anatomic cure rate of $80 \%(95 \%$ CI $75-86)$ is comparable with previous reports on midline fascial plication by other authors, though our study group was considerably larger $[4,5,15,16]$.

Only two factors, POP-Q stage $\geq$ III in the posterior compartment and a history of prior colposuspension, were identified as independent risk factors. Two other authors already earlier demonstrated that women with POP stage $\geq$ III are at increased risk of developing a recurrence or failure after surgical repair without grafts [17, 18]. Prior colposuspension is known to provoke posterior compartment prolapse, but has never been identified as a risk factor for recurrence after posterior compartment surgery [19].

An interesting finding was the fact that, at first glance, the anatomical outcome of patients with combined repairs appeared significantly better than the outcome after isolated repairs only. In the isolated repair group, though a significantly higher percentage of patients with a posterior POP stage III were present as compared with the combined repairs (Table 2). In the group that underwent concomitant repairs, deLancey's level I support was taken care of, as by the modified Manchester procedure or high McCall procedure, in 50 of 160 patients (31\%; Table 1) [20, 21]. It has been reported that apical support might explain half of the variation in anterior compartment support [22]. To a somewhat lesser extent, this could be demonstrated for the posterior compartment as well; in a group of patients with POP stage $\geq$ II Lowder et al. demonstrated that point Bp changed to stage 0 and I after simulated apical support in at least $30 \%$ of cases [8]. In our study though, we could not detect any significant protective effect of the above-mentioned apical support surgery on the anatomic outcome in the posterior compartment.

The vast majority of patients who underwent concomitant surgery underwent an anterior colporrhaphy as well (89\%). The success rate of the group as a whole in the anterior compartment was 61.5\% (54.4-67.7\%; Table 2). Although the outcome in the anterior compartment was not an endpoint in this study, results are half as good as compared with the posterior compartment, but comparable with recent reports by other authors [23, 24]. Concomitant anterior repairs did not influence outcome of the posterior compartment.

Although in our study, prior prolapse repair surgery, as a whole, appeared close to significance at univariate analysis; neither this factor nor a prior posterior wall repair could be identified as significant risk factor for anatomic failure of the posterior compartment. 
Functional results

All mean domain scores of the UDI show statistically significant improvements, of which two domains even with a large effect size, of which the domain of genital prolapse shows the largest calculated effect size at follow-up. Except for the domain of faecal incontinence, all domains of the DDI show statistically significant improvements as well, however, with a smaller effect size. Although only weak correlations between bowel symptoms and posterior vaginal wall prolapse have been reported, the defaecatory symptom that most consistently arose with respect to posterior vaginal prolapse was the need to splint the vagina or perineum to defaecate [25]. Thus, we were especially interested in the domain of obstructed defaecation. Seventy-five percent of patients were not considered to be significantly bothered by obstructed defaecation. That means that mean domain scores of the group as a whole are dampened by those who are not bothered at all, which is demonstrated by the low initial score. However, if patients were significantly bothered by obstructed defaecation, the improvement in this domain score appeared not only statistically significant, but also demonstrated a large effect size. In our study, patients that were significantly bothered by obstructed defaecation had a $63 \%$ chance that these symptoms improved or disappeared after surgery.

The positive effects of surgery on the quality of life of patients are particularly demonstrated for the domains of physical functioning and patient's mobility.

The percentage of patients that reported dyspareunia had decreased at follow-up.

Though $19 \%$ of patients reported de novo dyspareunia in $58 \%$, this complaint was no longer present after surgery. We realise that this section of the urogynecological questionnaire is rather intimate for most (older) patients and therefore the least well-answered part. However, the data are comparable with data published by other authors and earlier work by us [4, 15, 16, 26, 27].

Strengths and weaknesses

Strengths of this study are the large sample size with a high follow-up rate and the use of validated instruments as recommended by the ICS, such as POP-Q and validated urogynecological questionnaires. Another strength is the systematic surgical protocol followed by all surgeons.

Drawbacks, however, are that, at the start of our registry, we missed some questionnaires, so that the number of questionnaires at follow-up was somewhat higher than at baseline. The tendency of our, mostly older, patients to be somewhat reluctant with the response to questions on sexual functioning is another concern that deserves an appropriate solution for the benefit of future research.

\section{Conclusion}

Midline "fascial" plication under continuous digital transrectal control for the repair of symptomatic posterior vaginal wall prolapse is anatomically and functionally effective.

Two independent risk factors for anatomic failure could be identified: POP stage $\geq$ III of the posterior compartment and a history of prior colposuspension.

Acknowledgement Mr. Ruud van de Voorde for the medical photography.

\section{Conflicts of interest None.}

Open Access This article is distributed under the terms of the Creative Commons Attribution Noncommercial License which permits any noncommercial use, distribution, and reproduction in any medium, provided the original author(s) and source are credited.

\section{References}

1. Olsen AL, Smith VJ, Bergstrom JO, Colling JC, Clark AL (1997) Epidemiology of surgically managed pelvic organ prolapse and urinary incontinence. Obstet Gynecol 89:501-506

2. Maher C, Baessler K (2006) Surgical management of posterior vaginal wall prolapse an evidence-based literature review. Int Urogynecol J Pelvic Floor Dysfunct 17:84-88

3. Nieminen K, Hiltunen KM, Laitinen J, Oksala J, Heinonen PK (2004) Transanal or vaginal approach to rectocele repair: a prospective, randomized pilot study. Dis Colon Rectum 47:16361642

4. Maher CF, Qatawneh AM, Baessler K, Schluter PJ (2004) Midline rectovaginal fascial plication for repair of rectocele and obstructed defecation. Obstet Gynecol 104:685-689

5. Abramov Y, Gandhi S, Goldberg RP, Botros SM, Kwon C, Sand PK (2005) Site-specific rectocele repair compared with standard posterior colporrhaphy. Obstet Gynecol 105:314-318

6. Richardson AC (1993) The rectovaginal septum revisited: its relationship to rectocele and its importance in rectocele repair. Clin Obstet Gynecol 36:976-983

7. Miedel A, Tegerstedt G, Maehle-Schmidt M, Nyren O, Hammarstrom M (2008) Symptoms and pelvic support defects in specific compartments. Obstet Gynecol 112:851-858

8. Lowder JL, Park AJ, Ellison R, Ghetti C, Moalli P, Zyczynski H, Weber AM (2008) The role of apical vaginal support in the appearance of anterior and posterior vaginal prolapse. Obstet Gynecol 111:152-157

9. Bump RC, Mattiasson A, Bo K, Brubaker LP, DeLancey JO, Klarskov P, Shull BL, Smith AR (1996) The standardization of terminology of female pelvic organ prolapse and pelvic floor dysfunction. Am J Obstet Gynecol 175:10-17

10. van der Vaart CH, de Leeuw JR, Roovers JP, Heintz AP (2003) Measuring health-related quality of life in women with urogenital dysfunction: the urogenital distress inventory and incontinence impact questionnaire revisited. Neurourol Urodyn 22:97-104

11. van Brummen HJ, Bruinse HW, van de Pol G, Heintz AP, van der Vaart CH (2006) Defecatory symptoms during and after the first pregnancy: prevalences and associated factors. Int Urogynecol $\mathrm{J}$ Pelvic Floor Dysfunct 17:224-230 
12. Roovers JP, van der Bom JG, van der Vaart CH (2008) Hysterectomy does not cause constipation. Dis Colon Rectum 51:1068-1072, discussion 1072-1063

13. Kleeman SD, Karram M (2008) Posterior pelvic floor prolapse and a review of the anatomy, preoperative testing and surgical management. Minerva Ginecol 60:165-182

14. Cohen J (1988) Statistical power analysis for the behavioural sciences, 2nd edn. Erlbaum, Hillsdale NY

15. Paraiso MF, Barber MD, Muir TW, Walters MD (2006) Rectocele repair: a randomized trial of three surgical techniques including graft augmentation. Am J Obstet Gynecol 195: 1762-1771

16. Singh K, Cortes E, Reid WM (2003) Evaluation of the fascial technique for surgical repair of isolated posterior vaginal wall prolapse. Obstet Gynecol 101:320-324

17. Whiteside JL, Weber AM, Meyn LA, Walters MD (2004) Risk factors for prolapse recurrence after vaginal repair. Am J Obstet Gynecol 191:1533-1538

18. Salvatore S, Athanasiou S, Digesu GA, Soligo M, Sotiropoulou M, Serati M, Antsaklis A, Milani R (2009) Identification of risk factors for genital prolapse recurrence. Neurourol Urodyn 28:301-304

19. Kwon CH, Culligan PJ, Koduri S, Goldberg RP, Sand PK (2003) The development of pelvic organ prolapse following isolated Burch retropubic urethropexy. Int Urogynecol J Pelvic Floor Dysfunct 14:321-325, discussion 325

20. Goh JT, Tjandra JJ, Carey MP (2002) How could management of rectoceles be optimized? ANZ J Surg 72:896-901
21. Chene G, Tardieu AS, Savary D, Krief M, Boda C, Anton-Bousquet MC, Mansoor A (2008) Anatomical and functional results of McCall culdoplasty in the prevention of enteroceles and vaginal vault prolapse after vaginal hysterectomy. Int Urogynecol J Pelvic Floor Dysfunct 19:1007-1011

22. Summers A, Winkel LA, Hussain HK, DeLancey JO (2006) The relationship between anterior and apical compartment support. Am J Obstet Gynecol 194:1438-1443

23. Hiltunen R, Nieminen K, Takala T, Heiskanen E, Merikari M, Niemi K, Heinonen PK (2007) Low-weight polypropylene mesh for anterior vaginal wall prolapse: a randomized controlled trial. Obstet Gynecol 110:455-462

24. Nguyen JN, Burchette RJ (2008) Outcome after anterior vaginal prolapse repair: a randomized controlled trial. Obstet Gynecol 111:891-898

25. Jelovsek JE, Maher C, Barber MD (2007) Pelvic organ prolapse. Lancet 369:1027-1038

26. Stoutjesdijk JA, Vierhout ME, Spruijt JW, Massolt ET (2006) Does vaginal reconstructive surgery with or without vaginal hysterectomy or trachelectomy improve sexual well being? A prospective follow-up study. Int Urogynecol J Pelvic Floor Dysfunct 17:131-135

27. Milani AL, Heidema WM, van der Vloedt WS, Kluivers KB, Withagen MI, Vierhout ME (2008) Vaginal prolapse repair surgery augmented by ultra lightweight titanium coated polypropylene mesh. Eur J Obstet Gynecol Reprod Biol 138:232-238 